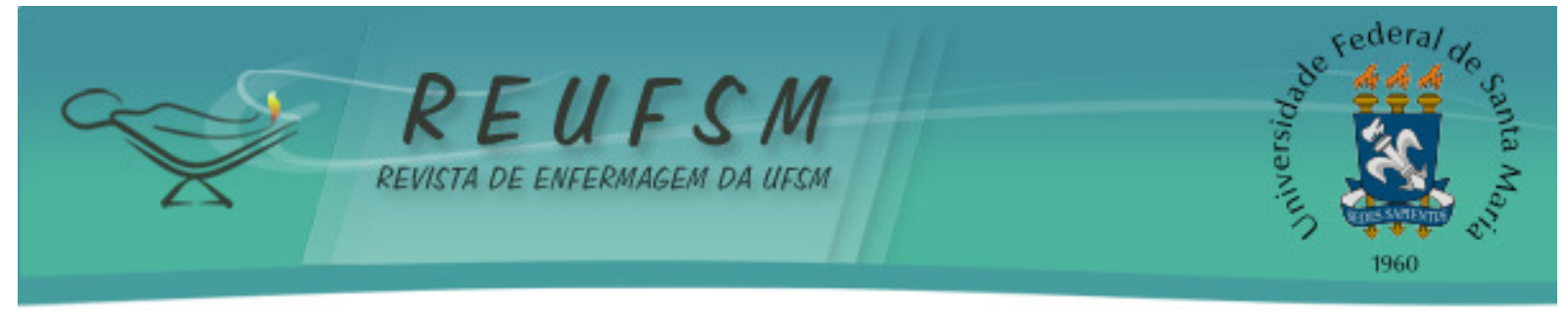

ARTIGO ORIGINAL

\title{
ADESÃO ÀS MEDIDAS DE PREVENÇÃO PARA PNEUMONIA ASSOCIADA À VENTILAÇÃO MECÂNICA
}

\section{ACCESSION TO THE PREVENTION MEASURES FOR PNEUMONIA ASSOCIATED WITH MECHANICAL VENTILATION}

\section{ADHESIÓN A LAS MEDIDAS DE PREVENCIÓN DE LA NEUMONÍA ASOCIADA A LAVENTILACIÓN MECÁNICA}

\author{
Kellyanny Maria Vasconcelos de Almeida ${ }^{1}$ \\ Ocilene Maria Cunha de Barros ${ }^{2}$ \\ Gabriela Jôse Candida Santos ${ }^{3}$ \\ Marilia Perrelli Valença ${ }^{4}$ \\ Aracele Tenório de Almeida Cavalcanti ${ }^{5}$ \\ Katia Oliveira Ferreira ${ }^{6}$
}

RESUMO: Objetivo: avaliar a adesão dos profissionais de saúde das unidades de terapia intensiva às medidas preventivas de pneumonia associada à ventilação mecânica implantadas no hospital. Método: estudo descritivo, quantitativo e observacional, desenvolvido com 130 pacientes em ventilação mecânica invasiva por mais de 48 horas, totalizando 481 observações, realizadas em um hospital universitário da cidade do Recife de fevereiro a abril de 2014. Resultados: os resultados revelaram fragilidade no cuidado e exposição dos pacientes a situações de risco, visto que, apenas 35,38\% dos pacientes acompanhados, aderiram completamente ao protocolo de prevenção de pneumonia associada à ventilação mecânica, sendo a interrupção da sedação e a higiene oral as intervenções que apresentaram o menor índice de conformidade, 70,06\% e 48,86\% respectivamente. Considerações finais: a qualidade do cuidado reflete na redução dos casos de pneumonia associada à ventilação mecânica e na segurança do paciente, demandando ações educativas multidisciplinares e auditorias periódicas.

Descritores: Infecção hospitalar; Pneumonia associada à ventilação mecânica; Unidades de terapia intensiva.

ABSTRACT: Aim: to assess adherence of health professionals from intensive care units concerning the preventive measures of ventilation associatedto pneumonia implemented in the hospital. Method: descriptive, quantitative and observational study, developed with 130 patients on mechanical ventilation for more than 48 hours, resulting in 481 observations, conducted at a university hospitalin Recife city fromfebruary to april 2014. Results:results revealed substandard care and patient exposure to hazards, since only

\footnotetext{
${ }^{1}$ Pós-Graduanda em Enfermagem em Unidade de Terapia Intensiva pelo Centro de Formação, aperfeiçoamento e Pesquisa-Cefapp. Recife, PE, Brasil. E-mail: kellyanny.kelly@gmail.com

2 Enfermeira. Recife, PE, Brasil. E-mail: celinhabarros@gmail.com

3 Pós-Graduanda de Enfermagem em Unidade de Terapia Intensiva pelo Centro de Formação, Aperfeiçoamento e Pesquisa-Cefapp. Recife, PE, Brasil. E-mail: gabi_jc@hotmail.com

4 Mestre em Ciências da Saúde- Faculdade de Ciências Médicas/UPE. Professora Assistente da Faculdade de Enfermagem Nossa Senhora das Graças - FENSG/ Universidade de Pernambuco. Recife, PE, Brasil. E-mail: mariliaperreli@gmail.com

5 Mestre em Ciências da Saúde pela Faculdade de Ciências Médicas - Universidade de Pernambuco. Recife, PE, Brasil. E-mail: aracelecavalcanti@hotmail.com

6 Enfermeira especialista em enfermagem do trabalho pela Estácio de Sá. Recife, PE, Brasil. E-mail: katiaferreira@yahoo.com.br
} 


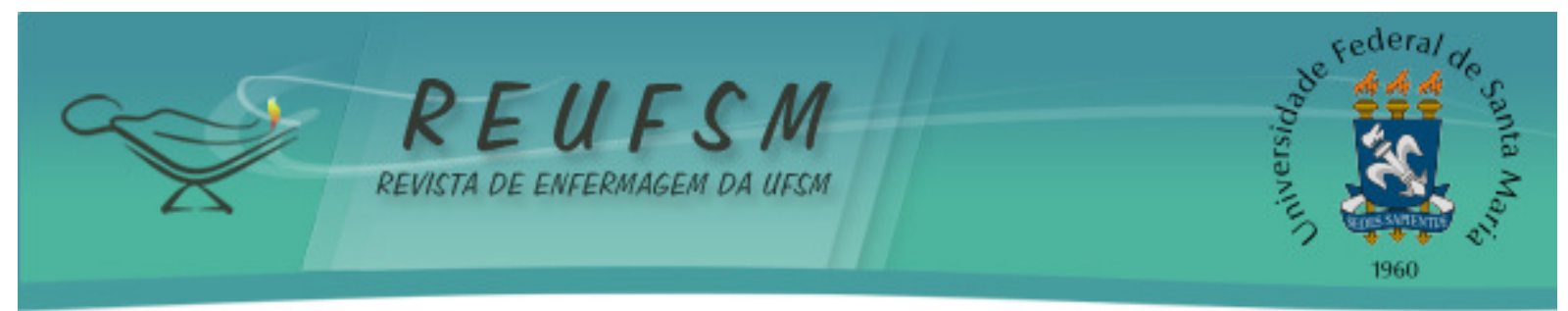

$35.38 \%$ of the patients, completely joined the ventilation associatedto pneumonia prevention protocol. Sedation interruptionand oral hygiene are the interventions that showed the lowest line index, $70.06 \%$ and $48.86 \%$ respectively. Final Remarks:quality care reflects in the reduction of pneumonia cases associated to mechanical ventilation and patient safety, therefore it requiresmultidisciplinary education and periodic lectures.

Descriptors: Infection control; Ventilator-associated pneumonia; Intensive care units.

RESUMEN: Objetivo: evaluar la adherencia de profesionales de la salud en unidades de cuidados intensivos a las medidas preventivas de la neumonía asociada a la ventilación mecánica implementada en el hospital. Método: estudio descriptivo, cuantitativo y observacional, desarrollado con 130 pacientes con ventilación mecánica invasiva durante más de 48 horas, con total de 481 observaciones, realizadas en un hospital universitario de la ciudad de Recife, de febrero-abril de 2014. Resultados: revelaron debilidad en el cuidado y exposición del paciente a riesgos, pues sólo $35,38 \%$ de los pacientes adherirán al protocolo de prevención de la neumonía asociada a ventilación, con interrupción de las intervenciones de sedación y de higiene oral que presentaron lo más bajo índice, $70.06 \%$ y 48.86\% respectivamente. Consideraciones finales: la calidad de la atención refleja en la reducción de neumonía asociada a la ventilación y en la seguridad del paciente, requiriendo prácticas educativas multidisciplinares y auditorías periódicas.

Descriptores: Control de Infecciones; Asociada a la ventilación neumonía; Unidades de cuidados intensivos.

\section{INTRODUÇÃO}

Com a introdução de novos métodos diagnósticos e terapêuticos, nas últimas décadas, observa-se um avanço tecnológico na assistência à saúde, com expressiva elevação na sobrevida da população. Entretanto, junto com a vantagem de prolongar a vida do paciente, esse quadro traz um aumento nos fatores que predispõem o surgimento das infecções hospitalares $(\mathrm{IH}){ }^{1}$

A IH, institucional ou nosocomial, segundo o Ministério da Saúde (MS), é qualquer infecção adquirida após a internação do paciente e que se manifesta durante sua permanência no hospital ou mesmo após a alta, uma vez que possa ser relacionada com a hospitalização, sendo esta apontada como um dos maiores riscos ao paciente. ${ }^{2}$

Dentre as IH destaca-se a pneumonia associada à ventilação mecânica (PAV), que é definida como uma infecção pulmonar que surge entre 48 à 72 horas após a intubação endotraqueal e a instituição da ventilação mecânica invasiva (VMI), como também até 48 horas após a extubação. É uma das IH mais incidentes nas Unidades de Terapia Intensiva (UTI), com taxas que variam de 9 a $40 \%$ das infecções adquiridas nesta unidade. Portanto, encontra-se como um dos eventos adversos mais temíveis no ambiente de terapia intensiva. ${ }^{3}$

Autores referem que a PAV apresenta uma mortalidade global que varia de 20 a $60 \%$, refletindo em grande parte a severidade da doença de base destes pacientes, a falência de órgãos, e especificidades da população estudada e do agente etiológico envolvido. Estimativas apontam que a mortalidade atribuída a esta infecção varia nos diferentes estudos, mas aproximadamente $33 \%$ dos pacientes com PAV morrem em decorrência direta desta infecção. ${ }^{4}$

A PAV é geralmente de origem aspirativa, sendo a principal fonte, as secreções das vias aéreas superiores, vindo a ocasionar uma resposta inflamatória do hospedeiro à multiplicação não controlada de microorganismos que invadem as vias aéreas distais. ${ }^{5-4}$ 


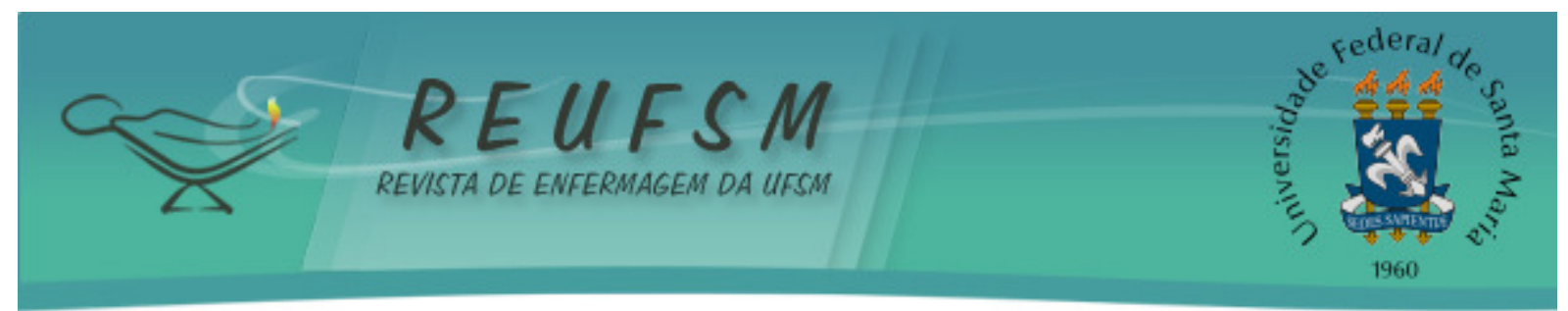

Estudos mostram que algumas condições relacionadas com a assistência à saúde são fatores de risco para a PAV, tais como aquelas que aumentam a colonização da orofaringe ou estômago por bactérias patogênicas, situações que causam a aspiração para o trato respiratório ou refluxo no trato gastrointestinal, uso prolongado da VMI e mãos contaminadas dos profissionais de saúde. ${ }^{6}$

Considerando a importância e a complexidade da PAV, faz-se necessário a realização de intervenções que causem impactos significativos à sua prevenção. Uma estratégia que tem sido adotada refere-se à criação de pacotes ou bundles de cuidados, os quais reúnem um pequeno grupo de intervenções, baseadas em evidências, que executadas coletivamente resultam em melhorias substanciais na assistência em saúde. ${ }^{7}$

Cabe salientar, que esses pacotes ou bundles de cuidados são aplicados de forma multidisciplinar e auditados pela Comissão de Controle de Infecção Hospitalar (CCIH), não vindo a exigir tecnologia complexa para sua realização, nem aumento na carga de trabalho dos profissionais ou custos adicionais para instituição, possibilitando sua aplicação em qualquer UTI. ${ }^{8}$

Nessa perspectiva, com intenção de realizar ações efetivas acerca da implantação das medidas de prevenção para PAV, torna-se válido questionar qual a adesão em unidades coronarianas de um Hospital Universitário para o bundle de PAV?

Diante do exposto, o referido estudo justifica-se pela grande relevância que a implantação do bundle traz para o cotidiano da instituição, vindo a contribuir com uma análise da adesão do mesmo pelos profissionais de saúde, fornecendo informações acerca da aplicação consistente dos cuidados e dados para possíveis intervenções.

Portanto, a adesão a estas medidas pode ser um poderoso instrumento para definição de estratégias voltadas a gerar impacto positivo na qualidade da assistência à saúde, e partindo desta premissa este estudo teve como objetivo avaliar a adesão do bundle de PAV em UTI.

\section{MÉTODO}

Trata-se de uma pesquisa de natureza descritiva, com abordagem quantitativa e observacional, desenvolvida nas Unidades Coronarianas I e II de um hospital universitário estadual, especializado em cardiologia na cidade do Recife-PE.

Foram incluídos no estudo todos os pacientes em VMI por mais de 48 horas que estavam internados nas Unidades Coronarianas (UCOs) do hospital em estudo, de ambos os sexos, no período de 26 de fevereiro a 30 de abril de 2014.

A coleta de dados foi realizada após a aprovação no Comitê de Ética em Pesquisa (CEP) sob o número do CAEE: 30541314.0.0000.5194, sendo respeitadas as diretrizes e normas recomendadas pela Resolução $n^{\circ} 466 / 12$ do Conselho Nacional de Saúde / MS, que regulamentam a pesquisa envolvendo seres humanos ${ }^{9}$.

Os dados utilizados para a elaboração da pesquisa foram coletados por meio de um check list elaborado pela $\mathrm{CCIH}$ do referido hospital, composto de perguntas objetivas acerca da realização dos cuidados contidos no bundle de PAV, sendo eles: elevação da cabeceira $30-45^{\circ}$, profilaxia de trombose venosa profunda (TVP), profilaxia de úlcera péptica, interrupção da sedação, higiene oral e pressão do cuff, as quais foram respondidas por meio da observação do paciente (elevação da cabeceira $30-45^{\circ}$ e interrupção da sedação) e por dados coletados no prontuário e nas prescrições do paciente (profilaxia de TVP, profilaxia de úlcera péptica, higiene oral e pressão do cuff), sendo preenchido pelas pesquisadoras diariamente de segunda à sexta-feira.

Cabe ressaltar que, em relação à mensuração da pressão do cuff, embora seja uma intervenção contida no bundle de PAV, configurou-se uma limitação para o estudo, pois, 


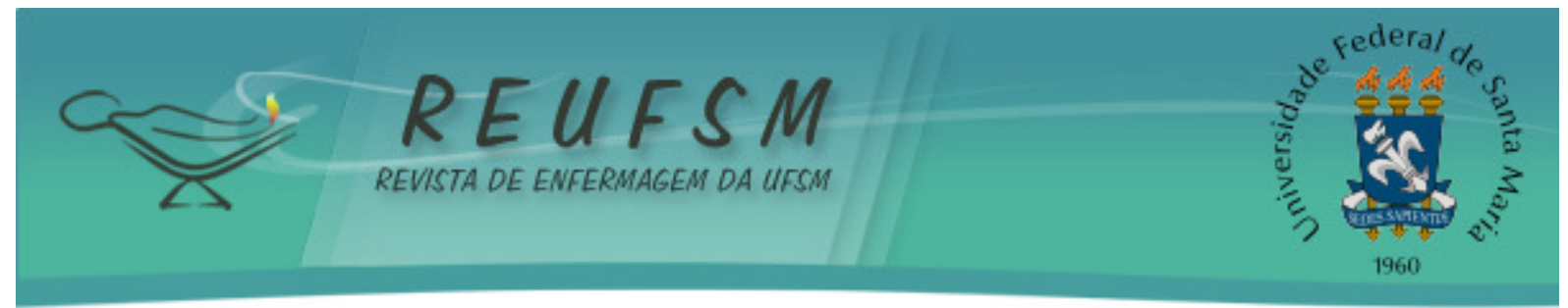

faltaram dados para avaliar esta intervenção, visto que, apesar da equipe de enfermagem e equipe médica estarem habilitadas para realizar esse cuidado, o mesmo era realizado apenas pelos fisioterapeutas, que na maioria das vezes não registram a mensuração do cuff na sua evolução.

A partir do levantamento das informações dos check list, utilizando-se do Epi Info 7, foi realizada uma análise estatística e descritiva a fim de estudar o comportamento das variáveis através do cálculo das frequência.

\section{RESULTADOS E DISCUSSÃO}

Foram acompanhados 130 pacientes em VMI que estiveram internados nas UCOs I e II no período 26 de fevereiro à 30 de abril de 2014, totalizando 46 dias de visitas, no qual foram realizadas no total 481 observações.

Tabela 1: Distribuição dos pacientes, segundo manutenção da cabeceira elevada à $30-45^{\circ}$. Hospital Universitário, Recife-PE, 2014.

$$
\text { Frequência } \%
$$

Elevação da Cabeceira 30-45 ${ }^{\circ}$

\begin{tabular}{lcc}
\hline Sim & 471 & $97,92 \%$ \\
Não & 10 & $2,08 \%$ \\
Total & 481 & $100 \%$ \\
\hline
\end{tabular}

Fonte: PROCAPE/RECIFE,2014

Em relação à manutenção da cabeceira elevada a $30-45^{\circ}$, das 481 observações, em 97,92\% (471) esta medida foi mantida, verificando-se que a mesma vem sendo bem difundida no referido hospital, evidenciado pelo bom índice de adesão dos profissionais de saúde.

A manutenção da cabeceira elevada a $30-45^{\circ}$ é uma medida relativamente simples e não demanda custos adicionais, sendo uma das principais recomendações para evitar broncoaspiração e, consequentemente a PAV, contribuindo para uma melhoria no volume corrente e ventilatório, vindo a diminuir inclusive os casos de atelectasia. ${ }^{10}$ Embora esta seja uma prática aparentemente simples, deve ser realizada com responsabilidade e valorizada pelos profissionais, pois configura um cuidado essencial na prevenção da PAV.

A profilaxia da TVP é uma medida indicada para indivíduos com 40 anos ou mais, com mobilidade reduzida e pelo menos um fator de risco adicional como os seguintes: acidente vascular encefálico (AVE), câncer, cateteres centrais e Swan-Ganz, doença inflamatória intestinal, doença respiratória grave, doença reumatológica aguda, gravidez e pós-parto, história prévia de TVP, infarto agudo do miocárdio, insuficiência cardíaca congestiva, infecção (exceto torácica), insuficiência arterial, internação em UTI, obesidade, paresia/paralisia MMII, quimio/hormonioterapia, reposição hormonal, síndrome nefrótica, trombofilia, exceto quando houver contraindicação. ${ }^{11}$ 


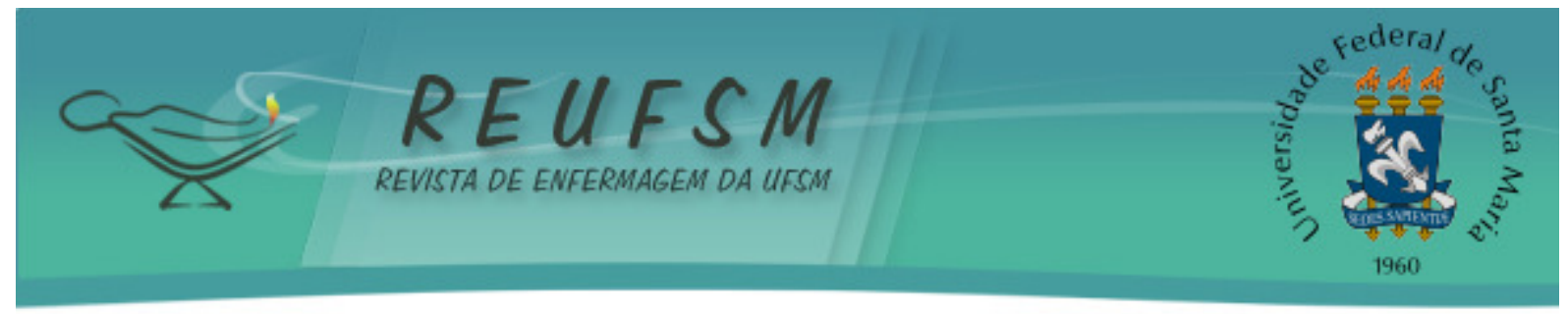

Tabela 2: Distribuição dos pacientes, segundo a profilaxia de TVP. Hospital Universitário, Recife-PE, 2014.

\begin{tabular}{lcc}
\hline & Frequência & $\%$ \\
\hline Profilaxia de TVP & & \\
\hline Sim & 321 & $66,67 \%$ \\
Não & 37 & $7,69 \%$ \\
Contraindicado & 123 & $25,57 \%$ \\
Total & 481 & $100,00 \%$ \\
\hline
\end{tabular}

Fonte: PROCAPE/RECIFE,2014

De acordo com a Tabela 2, das 481 prescrições verificadas, 66,74\% (321) continham medicamento profilático para TVP, 7,69\% (37) não constavam essa profilaxia, e 25,57\% (123) o paciente apresentava alguma contraindicação ao seu uso. Esse percentual foi significativo, revelando que a maioria dos profissionais aderiu a esta medida. 0 percentual de pacientes que não recebeu a profilaxia é pequeno, porém, recomenda-se, mesmo não sendo parte do bundle, instituir outras medidas de prevenção, como: uso de meia elástica, bota pneumática e exame físico dos MMll.

Embora a profilaxia de TVP não esteja associada diretamente à prevenção da PAV, sua prevenção tem impacto na diminuição da mortalidade hospitalar e na diminuição do tempo de internação, além disso, ressalta-se que as complicações da TVP retardam a extubação do paciente e aumentam o tempo de VMI, repercutindo em uma maior probabilidade para ocorrência da PAV.

Tabela 3: Distribuição dos pacientes, segundo a profilaxia de úlcera péptica. Hospital Universitário, Recife-PE, 2014.

\begin{tabular}{lcc}
\hline & Frequência & $\%$ \\
\hline Profilaxia de Úlcera Péptica & & \\
\hline Sim & 435 & $90,44 \%$ \\
Não & 36 & $7,48 \%$ \\
Contraindicado & 10 & $2,08 \%$ \\
Total & 481 & $100,00 \%$ \\
\hline
\end{tabular}

Fonte: PROCAPE/RECIFE,2014

Em estudo prospectivo de 2.252 pacientes críticos, encontraram incidência de Sangramento Digestivo por Ulcera de Estresse (SDUE) clinicamente importante de 1,50\%. Neste estudo, foram caracterizados como subgrupos de alto risco para SDUE, a necessidade de ventilação mecânica (VM) por mais de 48 horas (OD 15,6) e a presença de coagulopatia (OD 4,3). ${ }^{12}$ Desde então, ao reconhecer a VMI como um fator de risco para a formação de úlcera gástrica, algumas autores recomendam a adoção de medidas voltadas para evitar o aumento do $\mathrm{pH}$ estomacal. ${ }^{13}$

Nesse sentido, a profilaxia de úlcera péptica merece destaque como uma intervenção necessária a pacientes críticos. Assim, a Tabela 3, demonstra que das 481 prescrições analisadas, em 90,44\% (435) constavam essa profilaxia, em 7,38\% (36) não estava prescrito e em 2,08\% (10) o paciente apresentava alguma contraindicação, o que configura um bom percentual de adesão a esta medida. 


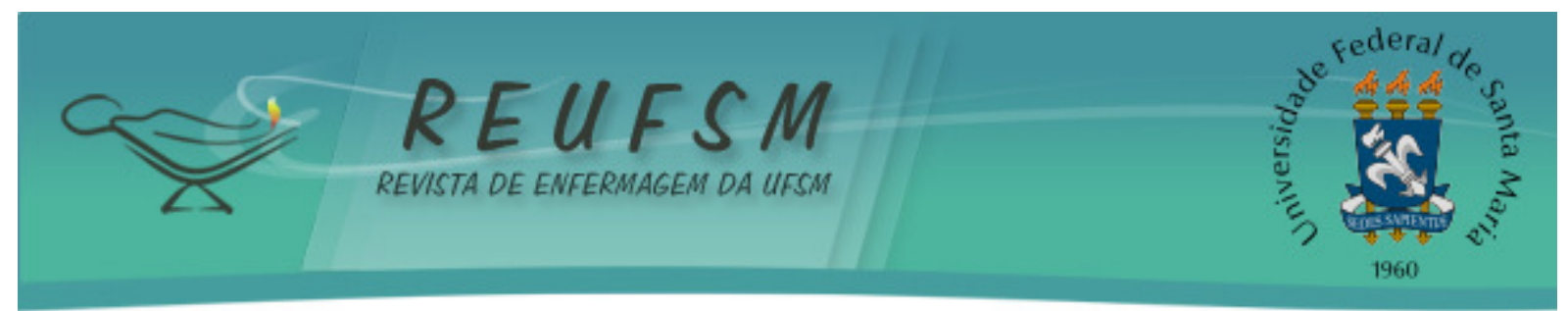

Cabe salientar, que a úlcera de estresse uma vez diagnosticada pode provocar hemorragia digestiva, piorar as condições clínicas do paciente e protelar sua saída da VMI, o que pode aumentar as chances de PAV, deixando clara a necessidade da sua profilaxia em pacientes críticos na UTI.

Porém, esta profilaxia gera questionamentos, devido seu potencial fator de risco para pneumonia nosocomial, pois agentes que elevam $\mathrm{o} \mathrm{pH}$ gástrico, alterando assim $\mathrm{o}$ padrão de colonização, podem promover o crescimento de bactérias no estômago, principalmente gram negativas originárias do duodeno. ${ }^{14}$

Outra preocupação importante é a relação entre a utilização de qualquer agente que suprima a acidez gástrica e o risco para o patógeno Clostridium difficile (C.difficile). ${ }^{7}$ Embora os esporos desse bacilo apresentem resistência ao $\mathrm{pH}$ ácido, as formas vegetativas sobrevivem melhor com o pH mais alcalino, sendo estas responsáveis por colonizarem a mucosa intestinal e produzirem as toxinas $A$ e $B$, responsáveis pela patogenia da doença $C$. difficile. ${ }^{15}$

Algumas pesquisas documentam este fato, entretanto, discute-se a intensidade do risco. Em uma recente meta-análise, após analisarem 42 estudos (30 caso-controle e 12 coorte), num total de 313 mil pacientes, encontraram um risco aumentado para infecção pelo Clostridium quando em usuários de inibidores da bomba de protóns, demonstrou- se uma ocorrência de 1,7 a 2,1 vezes maior de infecção pelo $C$. difficile. Refere-se ainda maior frequência de formas recorrentes nestes pacientes.

Portanto, torna-se necessário analisar o risco benefício dessa intervenção, de modo a garantir que a assistência recebida pelo paciente tenha um potencial maior de benefício que risco.

Tabela 4: Distribuição dos pacientes, segundo a interrupção da sedação. Hospital Universitário, Recife-PE, 2014.

\begin{tabular}{lcc}
\hline & Frequência & $\%$ \\
\hline Interrupção da Sedação & & \\
\hline Sim & 77 & $59,23 \%$ \\
Não & 53 & $40,77 \%$ \\
Total & 130 & $100,00 \%$ \\
\hline
\end{tabular}

Fonte: PROCAPE/RECIFE,2014

A administração contínua de sedativos é um preditor independente para maior duração da VMI, maior permanência na UTI e no hospital, e portanto, maiores chances de adquirir PAV. Sendo assim, a interrupção diária da sedação, assim como a avaliação da possibilidade de extubação dos pacientes, têm sido altamente recomendadas para prevenção da PAV, considerando que pacientes acordados podem contribuir no desmame ventilatório. $^{7}$

Nesse estudo observa-se, como apresentado na Tabela 4, que o protocolo para a interrupção da sedação foi realizado em $59,23 \%$ (77) dos pacientes. Constata-se, que $40,77 \%$ (53) dos pacientes que não tiveram a interrupção da sedação, não possuíam registro em prontuário que a justificasse.

Com a interrupção da sedação diariamente é possível avaliar o padrão respiratório do paciente e verificar se o mesmo pode ser extubado, com o intuito de reduzir o tempo de VMI e consequentemente o risco de PAV. Neste contexto, cabe à equipe multidisciplinar discutir quando será suspensa a sedação e ficar atenta às reações do paciente, devendo ainda monitorar o paciente para evitar a extubação acidental e quedas da cama ou maca. 


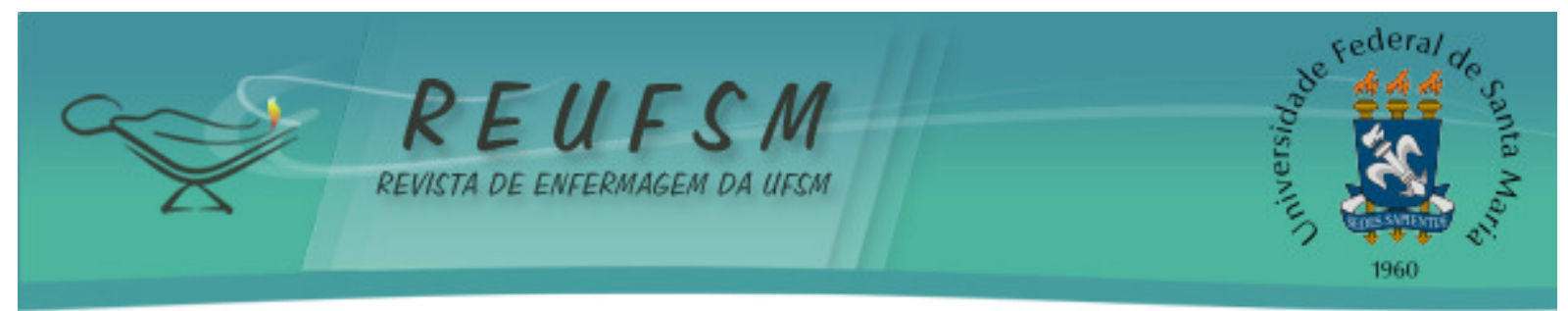

Um estudo sobre protocolo de sedação e interrupção diária da mesma trás em seus resultados que as principais razões que influenciam a baixa adesão a interrupção diária da sedação são a falta de aceitação da enfermagem, preocupação com o risco de remoção dos dispositivos pelo paciente e indução de outros comprometimentos respiratórios ou de desconforto para o paciente. ${ }^{16}$

Porém, é preciso ter cautela, pois apesar dos benefícios gerados pela interrupção diária da sedação, esta intervenção pode apresentar alguns riscos. 0 exemplo disso está na extubação acidental, no aumento do nível de dor e ansiedade e na possibilidade de assincronia com a ventilação, o que pode gerar períodos de dessaturação. ${ }^{7}$

Nessa perspectiva, cabe ao enfermeiro executar, coordenar e supervisionar as atividades ligadas a enfermagem, exigindo o preparo adequado, fortalecendo suas competências, atuando com objetividade em suas tarefas de orientar, interagir e avaliar, sendo o seu papel essencial na prevenção da PAV. ${ }^{17}$

Desse modo, a segurança do paciente emerge da interação dos componentes, conhecimento, habilidades, atitudes e responsabilidade no cuidar, onde todos que compõem a equipe multidisciplinar devem priorizar um tratamento benéfico à recuperação do paciente.

Outra importante medida na prevenção da PAV é a higienização adequada da cavidade oral do paciente em VMI, pois nesses casos há diminuição da produção de saliva e impossibilidade de mastigação, o que favorece o aparecimento de biofilme dental, sendo um importante reservatório para patógenos. ${ }^{7}$

Tabela 5: Distribuição dos pacientes segundo a higiene oral. Hospital Universitário, Recife-PE, 2014.

\begin{tabular}{lcc}
\hline & Frequência & $\%$ \\
\hline Higiene oral & & \\
\hline Sim & 235 & $48,86 \%$ \\
Não & 246 & $51,14 \%$ \\
Total & 481 & $100,00 \%$ \\
\hline
\end{tabular}

Fonte: PROCAPE/RECIFE,2014

O entendimento que a PAV é propiciada pela aspiração do conteúdo da orofaringe amparou a lógica de se tentar erradicar a colonização bacteriana desta topografia com o objetivo de reduzir a ocorrência da PAV. ${ }^{4}$

Nesse sentido, embora a Higiene Oral $(\mathrm{HO})$ caracterize um cuidado imprescindível em pacientes submetidos à VMI, os resultados obtidos no presente estudo, descritos na Tabela 5, demonstram uma baixa adesão, pois das 481 evoluções observadas na pesquisa, apenas 48,86\% (235) continham registros da realização da $\mathrm{HO}$, e $51,14 \%$ (246) não mencionavam este cuidado.

A cavidade bucal sofre colonização contínua, e a placa bacteriana serve de reservatório permanente de microorganismos, fator importante na fisiopatologia das pneumonias, principalmente quando o nível de consciência do paciente está rebaixado, pois essa condição favorece a microaspiração de secreções da orofaringe.

Estudo brasileiro sobre $\mathrm{HO}$ em UTI demonstra que o conhecimento dos profissionais de saúde sobre definiçãa, riscos e prevenção da PAV é regular, sendo a educação a melhor forma de se melhorar este conhecimento. ${ }^{18}$

Em relação à escassa documentação da $\mathrm{HO}$, cabe salientar que o registro de enfermagem ou anotação de enfermagem faz parte da comunicação escrita, sendo indispensável no cuidar, portanto, todas as informações fornecidas pelo paciente, 


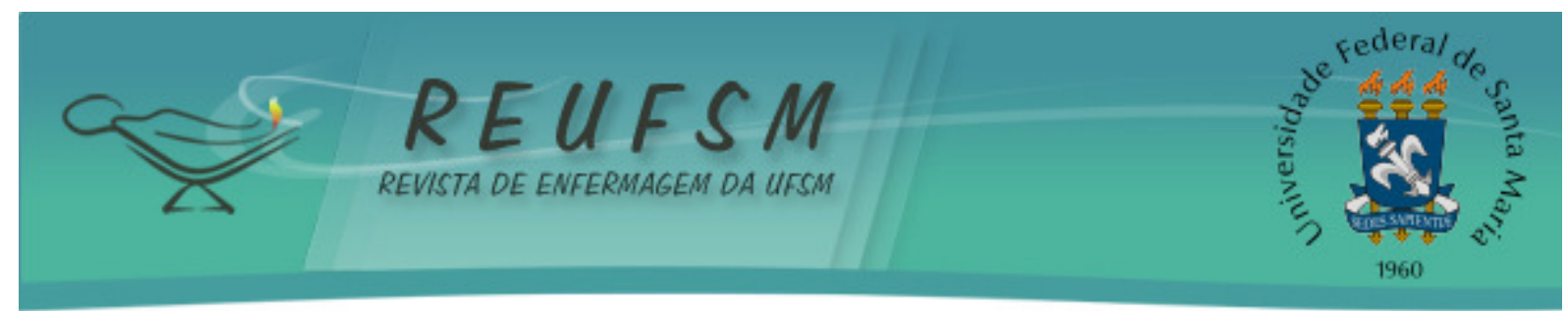

observações feitas pela equipe de enfermagem, assim como os cuidados prestados, devem ser registradas no prontuário do mesmo, de forma que toda a equipe tenha acesso, promovendo assim, um cuidado de forma integral, além de respaldo legal.

Portanto, sendo a $\mathrm{HO}$ um cuidado relevante na prevenção da PAV, é necessário buscar estimular a coparticipação e responsabilidade de todos os sujeitos envolvidos. Desta forma, a partilha do cuidado poderá contribuir para efetividade do processo do cuidar.

O bundle de PAV é uma série de intervenções que implementadas em conjunto resultarão em melhores resultados do que se realizadas individualmente.

Em relação à adesão do bundle por variável, observa-se que das cinco práticas analisadas, a interrupção da sedação e a $\mathrm{HO}$ foram as que obtiveram o menor índice de conformidade.

Nesse contexto, podemos citar como possíveis causas para não adesão a medida de $\mathrm{HO}$, a falta de conhecimento dos profissionais envolvidos quanto ao risco da não realização dessa medida, falta de conhecimento técnico para a realização deste ato, falta de capacitação em serviço, falta de uma sistematização da assistência de enfermagem e cuidados efetivos, ou até a ausência de uma auditoria diária, que cobre dos respectivos profissionais suas responsabilidades.

Cabe ressaltar que, a auditoria nesse contexto é considerada um elemento de mensuração da qualidade da assistência, oferecendo subsídios aos profissionais para (re)orientar suas atividades, estimulando a reflexão individual e coletiva norteando o processo de educação permanente. ${ }^{19}$

Em relação à interrupção da sedação, uma possível causa é a falta de um número de profissionais médicos, fisioterapeutas e de enfermagem adequados e bem treinados, falta de uma comunicação efetiva da equipe de saúde e de envolvimento da equipe multidisciplinar, visto que, trata-se de uma medida que exige vigilância contínua do paciente e detecção precoce da necessidade de retorno a sedação.

Torna-se válido ressaltar que para alcançar qualidade da assistência e segurança do paciente em VM com o uso do bundle é fundamental que todas as práticas estejam em conformidade, para que sua efetividade seja alcançada.

Destaca-se que, dos 130 pacientes acompanhados no período da pesquisa, apenas 35,38\% (46) apresentaram conformidade nas cinco intervenções analisadas no bundle, percentual que configura uma baixa adesão à realização simultânea dessas intervenções, vindo a comprometer a eficácia do bundle, pois, para que se obtenha sucesso na sua implementação não pode haver "mais-ou-menos", não há crédito parcial por fazer algumas das etapas. Os resultados são efetivos somente se todos os cuidados forem realizados em todos os momentos. ${ }^{7}$

\section{CONSIDERAÇÕES FINAIS}

Este estudo permitiu verificar a adesão dos profissionais de saúde de duas unidades coronarianas de um hospital universitário ao bundle de PAV, sendo este um conjunto pequeno e simples de práticas baseadas em evidências, que devem ser executadas coletivamente por todos que constituem a equipe multidisciplinar.

Cabe salientar que, para se alcançar qualidade da assistência e segurança do paciente em VM com o uso do bundle, é fundamental que todas as práticas estejam em conformidade, pois os resultados são efetivos somente se todos os cuidados forem realizados coletivamente durante toda permanência do paciente em VM.

Diante do exposto, considerando os objetivos do estudo, os resultados encontrados revelaram que das cinco práticas observadas no bundle, duas (profilaxia para TVP e HO) 


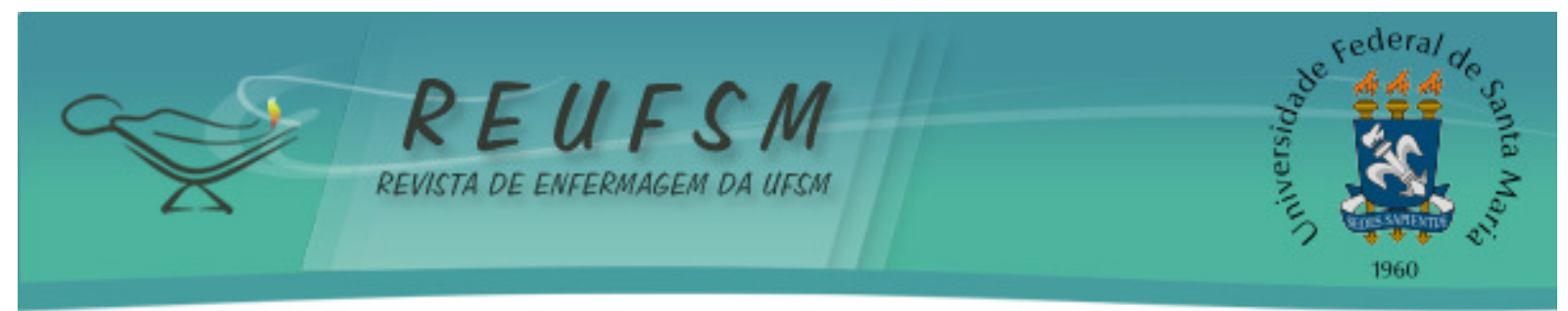

não apresentaram a conformidade esperada, portanto, as equipes multidisciplinares precisam intensificar os seus cuidados acerca das mesmas.

Esses resultados demonstram fragilidade do cuidado assistencial, e exposição dos pacientes a situações de risco para desenvolver PAV.

Por fim, a execução de medidas de prevenção baseadas em evidências científicas pode reduzir a PAV de forma significativa e sustentada, trazendo segurança na assistência e redução de custos, livrando o paciente de todas as implicações clínicas que uma infecção do trato respiratório hospitalar.

Assim sendo, acredita-se que esta pesquisa possa trazer subsídios para a melhoria da assistência prestada, por ter descrito a realidade da situação, atentando para necessidade de ações multidisciplinares que sejam aplicadas na prática diária da assistência prestada, assim como treinamentos e atualizações educativas em serviço e auditorias periódicas, pois práticas educativas de caráter permanente e contínuo configuram-se como ferramentas eficientes para o alcance da efetividade das práticas de prevenção.

\section{REFERÊNCIAS}

1. Guimarães AC, Donalisio MR, Santiago THR, Freire JB. Óbitos associados à infecção hospitalar, ocorridos em um hospital geral de Sumaré-SP, Brasil. Rev Bras Enferm [Internet]. 2011 [acesso em 2014 maio 3];64(5):864-9. Disponível em: http://www.scielo.br/pdf/reben/v64n5/a10v 64n5.pdf.

2. Brasil. Ministério da Saúde. Portaria $n^{\circ} 2616$, de 12 de maio de 1998. Expede em forma de anexos, diretrizes e normas para prevenção e o controle de infecções hospitalares. Diário Oficial da união, Brasília: DF; 1999.

3. Boundy J, Consendey $\mathrm{CH}$, Souza SR. Enfermagem médico-cirúrgica. $3^{\mathrm{a}}$ ed. Rio de janeiro: Reichmann e Affonso; 2009.

4. Brasil. Ministério da Saúde. Agência Nacional de Vigilância Sanitária (Anvisa). Critérios diagnósticos de infecção relacionada à assistência à saúde. $2^{a}$ ed. Brasília: Anvisa; 2013. (Segurança do Paciente e Qualidade em Serviços de Saúde).

5. Martins HS, Brandão Neto RA, Scalabrini Neto A, Velasco IT. Emergências clínicas: abordagem prática. 9ª ed. São Paulo: Manole; 2014.

6. Brasil. Ministério da Saúde. Agência Nacional de Vigilância Sanitária (ANVISA). Infecções do trato respiratório: orientações para prevenção de infecções relacionadas à assistência à saúde. Brasília: ANVISA; 2009.

7. Institute for Healthcare Improvement. 5 million lives campaign. Getting started kit: prevent ventilator-associated pneumonia: how-to guide. Cambridge, MA (US): Institute for Healthcare Improvement; 2010. 4 p.

8. Silva SG. Cuidado baseado em evidências: construção coletiva de um bundle de prevenção da pneumonia associada à ventilação mecânica [monografia]. Florianópolis, SC: Universidade Federal de Santa Catarina, Hospital Universitário Polydoro Ernani de São Thiago, Programa de Residência Integrada Multiprofissional em Saúde; 2012.

9. Brasil. Ministério da Saúde. Conselho Nacional de Saúde. Resolução CNS n 466, de 12 de dezembro de 2012. Aprovar as seguintes diretrizes e normas regulamentadoras de pesquisas envolvendo seres humanos. Brasília; 2012 [acesso em 2015 abr 6]. Disponível em: http://www.conselho.saude.gov.br/web_comissoes/conep/index.html. 


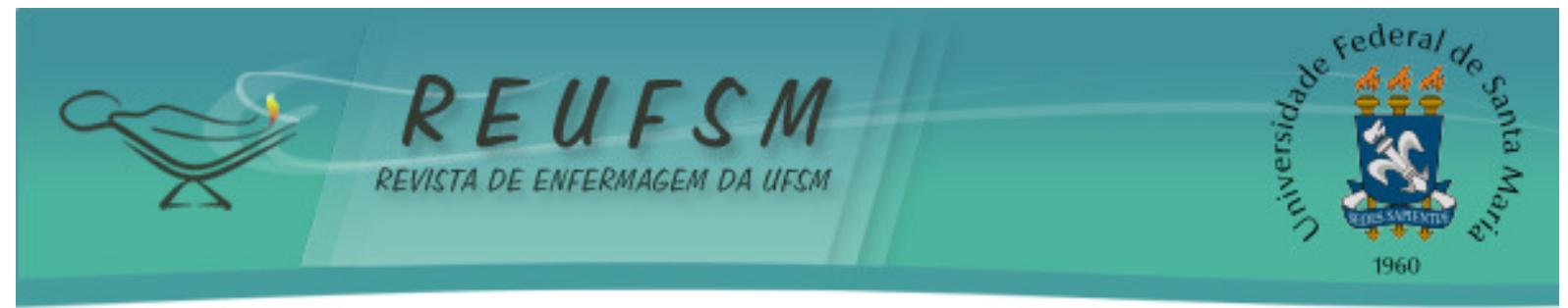

10. Bonten MJM. Ventilator-associated pneumonia: preventing the inevitable. Healthcare Epidemiology Clin Infect Dis [Internet]. 2011 jan [acesso em 2014 maio 2];52(1):115-121. Disponível em: http://cid.oxfordjournals.org/content/52/1/115.full.pdf.

11. Nacif SAP, Gazoni FM, Lopes RD. Profilaxia de tromboembolismo venoso em pacientes clínicos: como e quando? Rev Bras Clin Med [Internet]. 2009 jul [acesso em 2014 mar 2];7(5):331-8. Disponível em: http://files.bvs.br/upload/S/16791010/2009/v7n5/a011.pdf.

12. Cook DJ, Fuller HD, Guyatt GH, Marshall JC, Leasa D, Hall R, et al. Risk factors for gastrointestinal bleeding in critically ill patients. New Engl J Med. 1994;330(6):377-81.

13. Pruitt $B$, Jacobs $M$. Best pratice intervention: how can you prevent ventilatorassociated pneumonia? Nursing. $2006 \mathrm{fev}$;36(2):36-41.

14. Kusahara DM, Canezin CCS, Peterlini MAS, Pedreira MLG. Colonização e translocação bacteriana orofaríngea, gástrica e traqueal em crianças submetidas à ventilação pulmonar mecânica. Acta Paul Enferm [Internet]. 2012 [acesso em 2014 fev 1];25(3):398-400. Disponível em: http://www.scielo.br/pdf/ape/v25n3/v25n3a12.pdf.

15. Kwok CS, Arthur AK, Anibueze Cl, Singh S, Cavallazzi R, Loke YK. Risk of clostridium difficile infection with acid suppressing drugs and antibiotics: meta-analysis. Am J Gastroenterol. 2012;107(7):1011-9.

16. Tanios MA, Wit M, Epstein SK, Devlin JW. Perceived barriers to the use of sedation protocols and daily sedation interruption: a multidisciplinary survey. J Crit Care [Internet]. 2009 [acesso em 2014 maio 3];24(1):66-73. Disponível em: http: / / www.scielo.br/scielo.php?script=sci_nlinks\&pid=S0103507X20130 $0018600016 \&$ lng $=$ em.

17. Gomes AM, Silva RCL. Bundle de prevenção da pneumonia associada a ventilação mecânica: o que sabem os enfermeiros a esse respeito? [monografia]. Rio de Janeiro, RJ: Universidade Federal do Estado do Rio de Janeiro; 2009.

18. Silveira IR, Maia FOM, Gnatta JR, Lacerda RA. Higiene bucal: prática relevante na prevenção de pneumonia hospitalar em pacientes em estado crítico Acta Paul Enferm [Internet]. 2012 [acesso em 2014 abr 1];23(5):697-700. Disponível em: http://www.scielo.br/pdf/ape/v23n5/18.pdf.

19. Faraco MM, Albuquerque GL. Auditoria do método de assistência de enfermagem. Rev Bras Enferm [Internet]. 2004 jul-ago [acesso em 2015 fev 20];57(4):421-4. Disponível em: http://www.scielo.br/pdf/reben/v57n4/v57n4a07.pdf.

Data de recebimento: 08/09/2014

Data de aceite: 10/04/2015

Contato com autor responsável: Kellyanny Maria Vasconcelos de Almeida

Endereço postal:Avenida Tempo Feliz,150 Ap 101- Sancho

Cep: 50920-700 Recife-PE

E-mail:kellyanny.kelly@gmail.com 